\title{
Experiments to confront the environmental extremes of climate change
}

\author{
Zachary E Kayler ${ }^{1 *}$, Hans J De Boeck ${ }^{2}$, Simone Fatichi ${ }^{3}$, José M Grünzweig ${ }^{4}$, Lutz Merbold ${ }^{5}$, Claus Beier ${ }^{6}$, \\ Nathan McDowell ${ }^{7}$, and Jeffrey S Dukes ${ }^{8}$
}

\begin{abstract}
Extreme climate conditions can dramatically alter ecosystems and are expected to become more common in the future; however, our understanding of species and ecosystem responses to extreme conditions is limited. We must meet this challenge by designing experiments that cover broad ranges of environmental stress, extending to levels well beyond those observed currently. Such experiments are important because they can identify physiological, community, and biogeochemical thresholds, and improve our understanding of mechanistic ecological responses to climate extremes. Although natural environmental gradients can be used to observe a range of ecological responses, manipulation experiments - including those that impose drought and heat gradients - are necessary to induce variation beyond common limits. Importantly, manipulation experiments allow for determination of the cause and effect of species and ecosystem threshold responses. We present a rationale and recommendations for conducting extreme experiments that extend beyond the historical and even the predicted ranges of environmental conditions.
\end{abstract}

Front Ecol Environ 2015; 13(4): 219-225, doi:10.1890/140174

$\mathrm{C}$ limate extremes are expected to become more frequent and more intense, so that today's extremes may fall within normal background fluctuations in the future (Mora et al. 2013; Bahn et al. 2014). At the same time, new climate extremes will increase in magnitude and variability (Knapp et al. 2008). Extreme events can have important ecological consequences, as they can affect physiology and growth (Hueve et al. 2011), plant-insect interactions (McDowell et al. 2011), phenology and evolution (Gutschick and BassiriRad 2003), and soil carbon (C) exchange (Sowerby et al. 2008). Smith (2011) defined a climate extreme as a statistically rare event that can "alter ecosystem structure and/or function well outside the bounds of what is considered typical or normal variability". Importantly, climate

\section{In a nutshell:}

- Climate extremes are known to alter ecosystem function and structure

- Few experiments have intentionally exceeded known biological and biogeochemical tolerances to climatic extremes

- We advocate experimental designs that impose wide stress gradients that are designed to identify response thresholds

- Such experiments will guide mechanistic models, with the ultimate aim of projecting when climate extremes will provoke non-linear responses or cause ecosystems to pass tipping points

${ }^{1}$ Institute for Landscape Biogeochemistry, Leibniz Center for Agricultural Landscape Research (ZALF), Müncheberg, Germany "(kayler@zalf.de); ${ }^{2}$ Research Group of Plant and Vegetation Ecology, Department of Biology, Universiteit Antwerpen, Wilrijk, Belgium; ${ }^{3}$ Institute of Environmental Engineering, ETH Zurich, Zurich, Switzerland; continued on p 225 extremes do not always lead to ecological extremes, because many species have evolved to tolerate or be resilient to a broad range of stressors (Scheffer and Carpenter 2003; Smith 2011; Hoover et al. 2014).

Currently, little is known about how species and ecosystems respond when they are pushed beyond the environmental and biological conditions under which they have evolved. However, ongoing environmental disruptions, such as climate change, increase the probability that ecosystems will eventually experience conditions currently deemed "unrealistic". Without an understanding of the mechanisms and limits of resilience for species and ecosystems, researchers will be unable to predict when thresholds for critical ecosystem functions might be passed (Lenton 2011). Experiments are needed that will push species and ecosystems past environmental thresholds to provide insights into mechanistic functions and broaden the boundaries of environmental conditions for which we can make predictions and extrapolate results to larger spatial and temporal scales (Figure 1).

Few ecological studies have "pushed the envelope" in terms of experimental criteria for climate research, for a number of possible reasons: many researchers (1) are not aware of the critical role extremes play in a changing climate; (2) may be wary of causing permanent damage to their long-term studies; (3) are concerned about their study being branded as unrealistic and therefore unpublishable; (4) believe there are technical limitations to imposing "extreme" conditions; and (5) are faced with legal restrictions that prevent extreme manipulations in natural areas. We present a rationale for conducting manipulation experiments (Figure 2) that exceed the historical and even the predicted range of environmental conditions. We contend that such studies provide the most viable means of identify- 


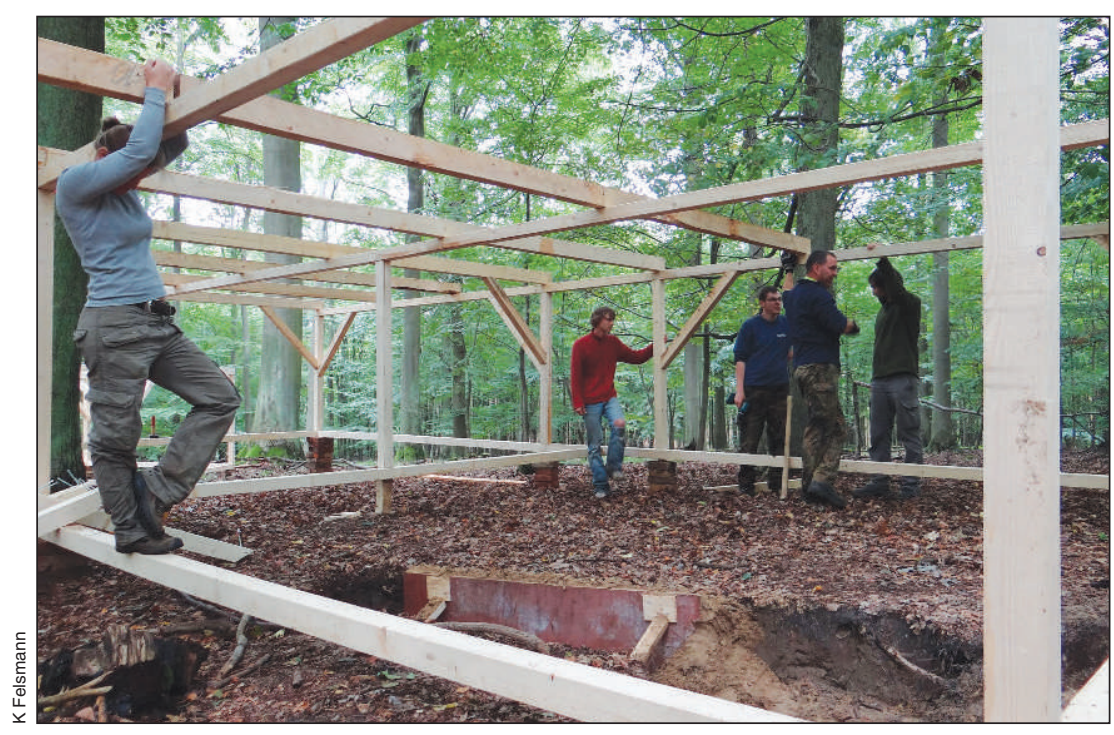

Figure 1. Construction of a series of roofs used at a rainfall reduction site. In the project, called BE-Dry, roofs were erected in forests in Germany to manipulate soil water levels. The interactions between plant and microbial diversity, and subsequent feedbacks to soil physical and hydrological properties, were observed over the growing season.

ing critical thresholds in species survival and ecosystem functioning, as well as the corresponding threshold mechanisms. We describe several gradient experiments that included climate extremes that have improved the mechanistic understanding of important ecosystem processes such as productivity, biogeochemical cycles, and stress tolerance. We also present guidelines for conducting such manipulation experiments, highlighting the importance of coordinating with members of the modeling community.

\section{Natural gradients and manipulations}

Observations from persistent natural stress gradients (eg carbon dioxide $\left[\mathrm{CO}_{2}\right]$ springs, thermal gradients) are an

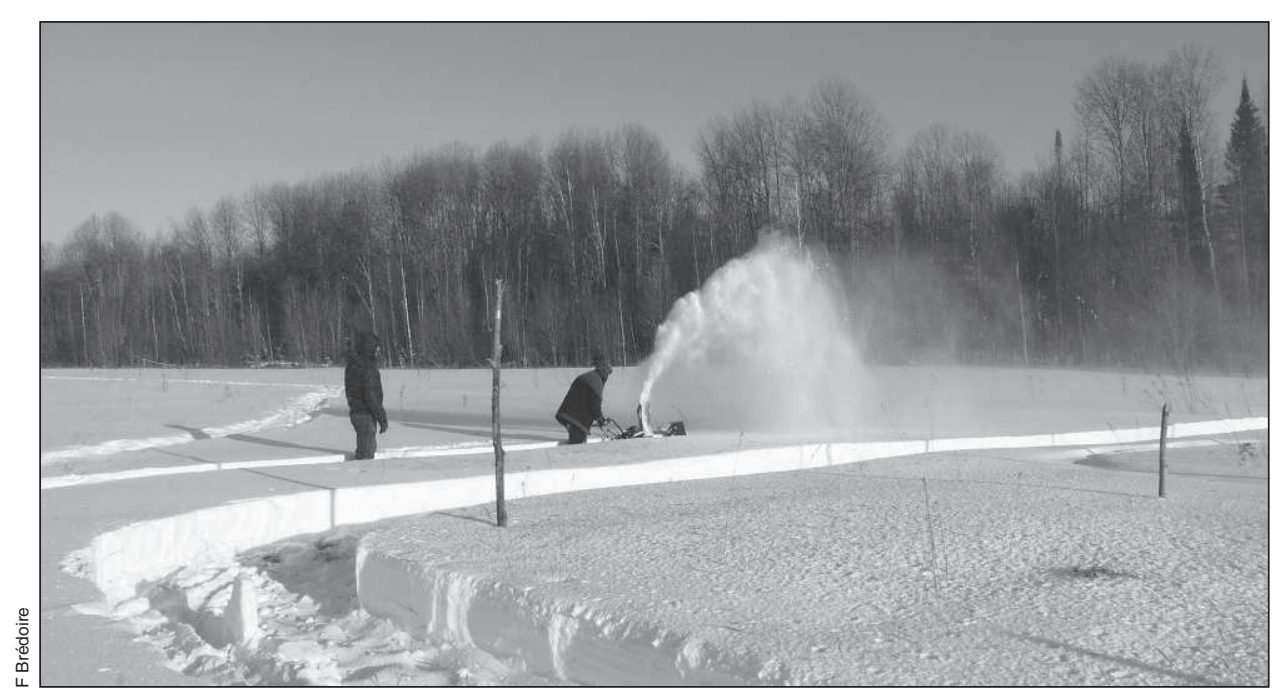

Figure 2. The beginning of a snow manipulation experiment in southwestern Siberia. Snow levels were doubled in an experiment designed to investigate the impact of a deeper snowpack on nitrogen and water biogeochemistry in agricultural sites near Barnaul, Russia. important source of information for extreme-environments research and have provided initial insights into possible community, physiological, and biogeochemical responses to extreme changes in climate (Sagarin and Pauchard 2009). Sites where such gradients are present provide an opportunity to study long-term response patterns using natural archives such as tree rings, as well as short-term effects when experiments are initiated soon after system-altering events occur.

Geological $\mathrm{CO}_{2}$ springs provide a natural gradient of atmospheric concentrations that is extreme in terms of both magnitude and duration; the study of such springs has improved our understanding of physiological responses, including acclimation of plants and ecosystems to high concentrations of $\mathrm{CO}_{2}$. Vegetation that grows near these natural springs can experience $\mathrm{CO}_{2}$ concentrations greater than 1000 parts per million near the source, decreasing gradually over hundreds of meters (Hättenschwiler et al. 1997; Tognetti et al. 2000). By comparing plant physiology and multidecadal stem growth of trees located near and far from $\mathrm{CO}_{2}$ springs in the Mediterranean region, studies have revealed a weak or null effect of elevated $\mathrm{CO}_{2}$ concentrations on tree growth, despite persistent effects on stomatal functioning (Hättenschwiler et al. 1997; Tognetti et al. 2000).

A new research project in Iceland (Figure 3 ) takes advantage of natural increases in soil temperature caused by volcanic activity to study ecosystem responses to soil warming. Such "hotspots" can relocate over time, potentially enabling researchers to look into both short-term $(\sim 5$ years) and long-term ( $>30$ years) effects. The project is investigating both normal temperature gradients of only a few degrees Celsius and the effects of much greater warming $\left(+10^{\circ} \mathrm{C}\right.$ and higher) to detect thresholds and optima for different processes. Researchers are measuring a variety of biogeochemical and phenological parameters, such as decomposition rates, nitrous oxide $\left(\mathrm{N}_{2} \mathrm{O}\right)$ fluxes, and radial tree growth. Particular emphasis is placed on processes that are sensitive to low temperature thresholds, as well as others that appear to share threshold levels (eg plant activity and mycorrhizal associations), with the aim of identifying possible linkages between them. 
Although studies conducted along such natural gradients provide opportunities to examine long-term responses to specific drivers across a wide range of conditions, manipulation experiments that artificially impose extreme conditions allow researchers to isolate mechanisms and identify response thresholds (Figure 4). Previous experiments of this kind have yielded valuable information about biological and biogeochemical responses, including the responses of plants exposed to elevated ultraviolet radiation (Paul 2000) and ecosystems subjected to a gradient from preindustrial to high future $\mathrm{CO}_{2}$ concentrations (Grünzweig and Körner 2001). Here, we focus on a few particularly successful examples of such manipulations.

Hoover et al. (2014) performed an ecosystem-level manipulation experiment that tested the effects of extreme drought and short-term heating on the annual aboveground net primary production (ANPP) of grasslands. Using long-

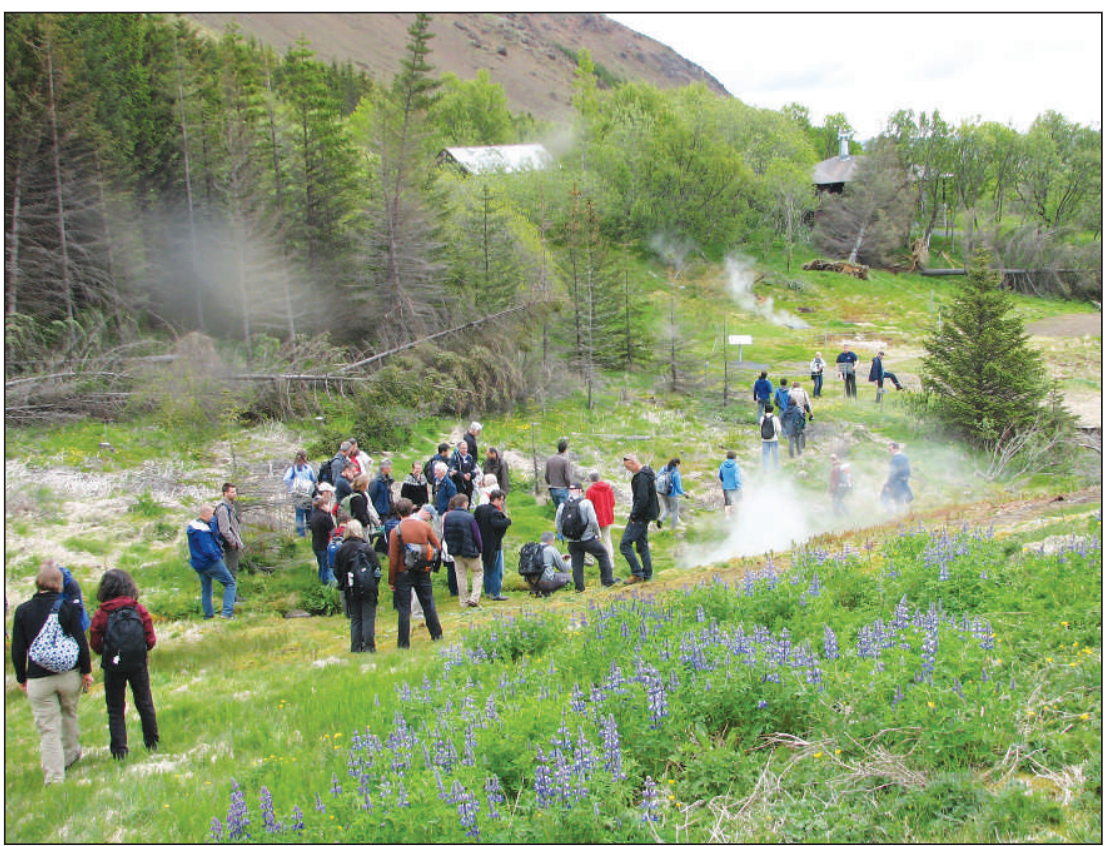

Figure 3. Site of a new project (FORHOT) in Iceland. Researchers measure a variety of biogeochemical and phenological parameters, such as decomposition rates, $\mathrm{N}_{2} \mathrm{O}$ fluxes, and radial tree growth. This project is a prime example of using natural gradients to study environmental extremes. term climate data, they were able to determine extreme environmental conditions for both precipitation and temperature before the experiment began. Grassland ANPP was significantly reduced as a result of drought, whereas temperature did not have a detectable direct effect, demonstrating that imposed extremes can lead to different ecological responses (Smith 2011). Interestingly, the authors also found that the grassland in question achieved pre-treatment ANPP levels during a recovery period in the following growing season. They attributed this to a shift in dominant species, which compensated for the loss of production associated with the former dominant species. This study showed how manipulation experiments can be used to understand the mechanisms behind community responses to climate extremes and how these responses manifest themselves in ecosystem function - in this case in ecosystem resilience.

Two studies that examined the mechanisms of droughtinduced tree mortality (Hartmann et al. 2013; Sevanto et al. 2014) provide an example of an innovative manipulation experiment that pushed organisms to physiological extremes. The authors tested the role of $\mathrm{C}$ starvation (when metabolic demand outweighs available C) versus hydraulic failure (when plant water and transport levels decrease below a threshold that supports cellular function; McDowell 2011). The physiological responses of the trees to drought and $\mathrm{C}$ starvation, as well as the effects on hydraulic transport and carbohydrate status, were tracked until tree death. Ultimately, these studies concluded that trees can withstand a low C supply far longer than they can endure persistent drought conditions. These studies highlight the potential benefits of using extreme treatment levels; they identified both gradual responses that corresponded to drivers $\left(\mathrm{CO}_{2}\right.$ levels and drought stress) as well as an abrupt response (ie leaf water potential in Sevanto et al. [2014]) corresponding to a physiological threshold.

\section{Ideas for the future}

The goal of the experiments proposed here is to establish physiological, phenological, community, and biogeochemical thresholds by assessing organism, community, and ecosystem responses along natural or experimental gradients that span a broad range of ecological conditions, including extremes. To accomplish this, scientists must move away from conducting experiments using the conventional, single-treatment-level-plus-control approach and instead evaluate - in real time - physiological and biogeochemical responses to treatments that extend beyond normal levels (Bahn et al. 2014). Researchers need to find ways to overcome the technical limitations that often curtail extreme manipulations. These experiments should include a modeling strategy before the experiment, during the planning phase, and after the experiment for analyzing the results and assessing the models used to generalize the processes under investigation. Our general understanding of responses to extreme conditions will be further improved if researchers conduct coordinated measurements that are comparable across systems, enabling cross-site analyses, similar to what has already been established for flux data (Baldocchi et al. 1996).

A stress gradient imposed through discrete steps will help to identify non-linearities and the thresholds of 


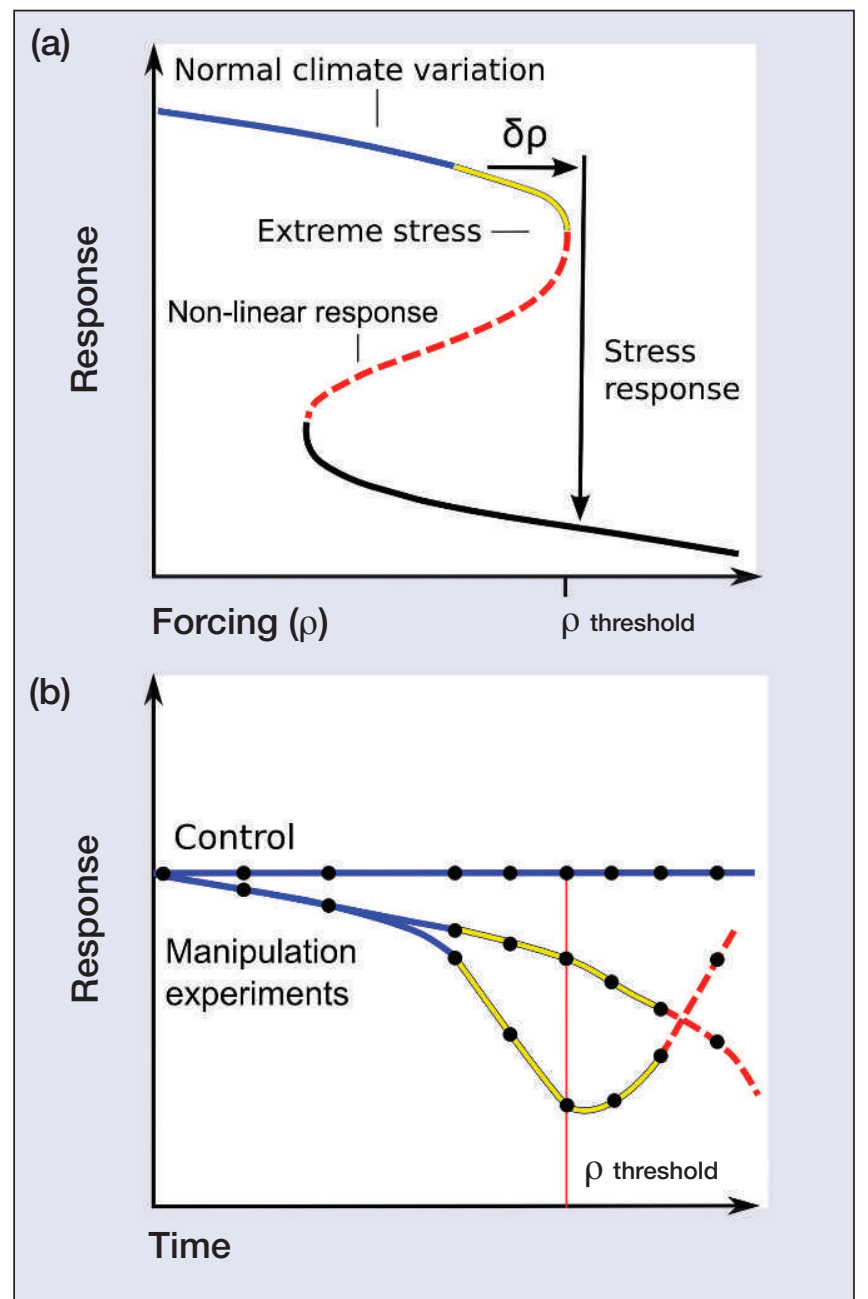

ecosystem responses, which are otherwise difficult to detect through single-treatment experiments. Previous manipulation experiments have applied one or more levels of environmental stress but only a few have applied treatments that produce strong gradients of different conditions (Hoeppner and Dukes 2012). The capacity to iteratively adjust treatments to achieve a desired range of physiological and biogeochemical responses (ie responsedriven treatment levels) is one advantage of studying responses along a gradient. This optimization can, in turn, lead to capturing a threshold response resulting from an incremental shift in a treatment level, thus providing an endpoint to the expected biological or biogeochemical response (Figure 4). From such experiments that include climate extremes with regards to both the overall treatment mean values and the degree of variability, we can then quantify unexpected multiple non-linear responses. Ultimately, these results can be used to better represent biological responses to extreme stress levels and improve model predictions.

Thresholds can be expressed at different levels of organization. For example, at the organismal level, thresholds may not be apparent until death (eg in the case of water tension in drought-stressed trees; Sevanto et al. 2014) or dormancy in the case of microbes (Jones and Lennon 2010). Approaches from other disciplines, such
Figure 4. Conceptual model of thresholds related to environmental stress $(\rho)$ and the subsequent system response ([a]; adapted from Lenton 2011), and how these are linked to possible responses to extreme climate experiments (b). The different colors along the response trajectory refer to normal climate variations that are the focus of monitoring or conventional experiments (blue), extreme stress experiments (yellow), and non-linear responses of the system or possible death of the study organism (red). The line following the non-linear response (black) indicates a new system state or structure, which may or may not be included in the manipulation experiment. The same color scheme holds for the bottom panel in which three example experiments are illustrated (one control and two treatments). If experiments were tailored solely for recent climate conditions (blue), then the experiment might miss threshold behavior and the uncertainty in response trajectory once the threshold stress level is passed. Black circles represent sampling points along an imposed stress gradient, which we argue is a clear strategy for capturing biological and biogeochemical thresholds, in contrast to experiments that consider only one stress level.

as "lethal dose" (LD), can be used to describe species' thresholds in climate-change studies. For instance, Larcher et al. (2010) looked at levels of damaged plant tissue (identifying the LD to $0 \%, 50 \%$, and $100 \%$ of plant test subjects) associated with frost stress to understand alpine plant survival under extreme temperatures. At the community level, thresholds can be identified through changes in community composition that cascade through the ecosystem, altering its structure. These ecological response variables include changes in resilience, disturbance regimes, and invasibility, as well as changes in biophysical attributes, such as canopy height or soil hydrophobicity (Figure 5). Furthermore, how changes in communities and organisms manifest in biogeochemical cycles or emergent properties, such as diversity, is relevant to understanding extreme events (Figure 6); Fuchslueger et al. (2014) used stable isotopes to investigate meadow ecosystem responses to extended drought, observing that the drought induced changes in the active microbial communities. The authors found that drought plots had greater microbial biomass but received less of the labeled $\mathrm{C}$ isotope, illustrating the complexity of the relationship between community dynamics and biogeochemical cycles.

\section{Models: a tool for experimental design and analysis}

Models synthesize the current mechanistic and conceptual understanding of ecosystems and can be useful at different stages of the proposed manipulation experiments. For example, at the design stage, models can help predict the stress level associated with a physiological or biogeochemical threshold response. Conversely, experimental results can be used to validate current modeling assumptions concerning mechanisms of ecosystem response under extreme stress. In this section, we briefly discuss 
the benefits and limitations of using models as tools for experimental design and post-experiment synthesis.

To guide the design of these experiments, we recommend using mechanistic models that simulate different dynamics of vegetation and/or biogeochemistry and their responses to external forcings. Mechanistic models allow researchers to speculate beforehand about the magnitude of responses to the applied treatments and can guide decisions regarding monitoring additional variables or states that have not previously been considered. However, particularly during the design of extreme manipulations, the results from mechanistic models should be regarded as a supporting tool and should be viewed with caution. This is largely because model simulations can differ greatly from the measured responses after the experiment, especially when ecosystems experience unprecedented conditions. Abrupt responses and "breaking points" (eg

Figure 4a) in models can originate from (1) parameterized thresholds for environmental variables (eg minimum temperature for survivorship, maximum water potential for cavitation) or (2) a combination of multiple non-linear processes (eg $\mathrm{C}$ starvation, species disappearance). In the first case, models are unlikely to provide new insights, while in the second case, models should be able to integrate processes that are individually understood and reproduce emergent and non-linear responses. If the model cannot capture complex ecosystem responses, then the mechanistic representations of one or more of the individual processes are not completely understood and will require further investigation.

Ultimately, models can be used to help interpret experimental results, which should, in turn, inform model structure (Beier 2004). A model that is able to capture the essential effects of the experimental treatment without fundamentally changing the model - will demonstrate a robust predictive capability (at least for the specific treatment applied). Despite the growing number of environmental manipulation experiments, few modeling studies have compared the performance of multiple models in reproducing treatment conditions (eg Fatichi and Leuzinger 2013; McDowell et al. 2013). Such comparisons have great value, as they can provide fundamental tests of the predictive capabilities of models.

\section{General guidelines}

We recommend the following general elements for experiments designed to determine thresholds at the organism, community, and ecosystem level. As emphasized by Smith (2011), knowledge of preexisting environmental conditions is necessary to consider the breadth of the stress gradient (eg different colors in Figure 4a) to be imposed. Mechanistic models can be used beforehand to avoid treatments for which responses are unlikely to be detected due to limitations in accuracy and precision of the planned measurements and a limited number of replicates. During the experiment, different measurements of ecological responses in process, structure, and function (Figure 5) should be made along the gradient (Figure 4b). For example, canopy wilting is a simple observation that can help identify a moisture stress threshold that may be complemented by continuous process measurements such as plant transpiration and soil water potential; additional examples are provided in Figure 5. The level of biological organization must also be considered, as climate extremes can lead to changes in dominant species (Allen and Breshears 1998), which, in turn, may substantially alter ecosystem functioning (although not necessarily; see Hoover et al. 2014). As a final step, experimental results can be used with models to optimize how biological processes are represented within the model.

\section{Conclusions}

Applying manipulation experiments across wide treatment ranges - even those considered unrealistic - will help to characterize the full spectrum of responses to climate extremes and will bring the field of ecology a major step forward in predicting ecosystem responses to future climate scenarios (Knapp et al. 2008). Previous studies, using naturally occurring or intentionally extreme treatments, have paved the way for the implementation of this strategy. Our recommendations - applying a gradient 


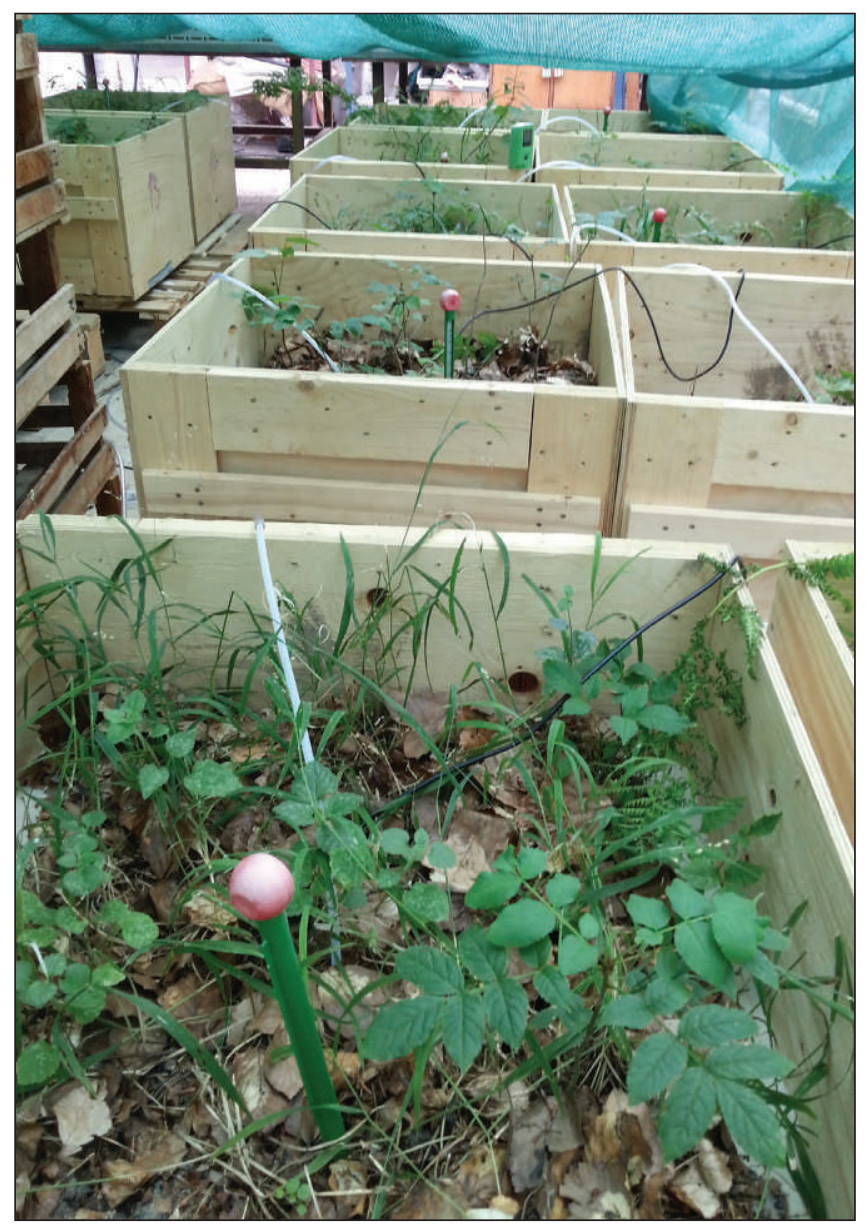

Figure 6. Soil monoliths before a drought and heat experiment. Intact soil monoliths were removed from a beech (Fagus sylvatica) forest in Germany to impose a drought gradient and heat pulse treatment that included extremes. The experiment is designed to investigate the $\mathrm{C}$ continuum of plants and soil, including the response of belowground biogeochemistry and microbial communities.

approach including extreme conditions, establishing a common measurement platform, and modeling both before and after experiments - are intended to refine this strategy.

Risk is inherent in such experiments, since permanent damage to the subjects is to be expected when they are exposed to the most extreme conditions. Moreover, there are many technical challenges involved in detecting incremental changes in subject responses. Yet high-risk endeavors can often lead to greater payoffs and new discoveries. Implementing the proposed experiments will advance our mechanistic and conceptual understanding of ecosystem function, which is especially relevant given the accelerating rate of climate change.

\section{Acknowledgements}

This paper resulted from discussions held during the Open Science Conference on Climate Extremes and Biogeochemical Cycles in the Terrestrial Biosphere:
Impacts and Feedbacks Across Scales, in Seefeld, Austria. The meeting was sponsored by CARBO-Extreme (FP7 2007-2013, grant agreement 226701), the Integrated Network for Terrestrial Ecosystem Research on Feedbacks to the Atmosphere and Climate (INTERFACE) (NSF DEB-0955771), and the Integrated Land EcosystemAtmosphere Processes Study (iLEAPS). CB was supported by the VELUX Foundation through the CLIMAITE project. HJDB is a post-doctoral researcher of the Fund for Scientific Research - Flanders. We thank N Glæsner for incisive comments on an earlier version of this manuscript.

\section{References}

Allen CD and Breshears DD. 1998. Drought-induced shift of a forest-woodland ecotone: rapid landscape response to climate variation. P Natl Acad Sci USA 95: 14839-42.

Bahn M, Reichstein M, Dukes JS, et al. 2014. Climate-biosphere interactions in a more extreme world. New Phytol 202: 356-59.

Baldocchi D, Valentini R, Running S, et al. 1996. Strategies for measuring and modelling carbon dioxide and water vapor fluxes over terrestrial ecosystems. Global Change Biol 2: 159-68.

Beier C. 2004. Climate change and ecosystem function - full-scale manipulations of $\mathrm{CO}_{2}$ and temperature. New Phytol 162: 243-45.

Fatichi S and Leuzinger S. 2013. Reconciling observations with modeling: the fate of water and carbon allocation in a mature deciduous forest exposed to elevated $\mathrm{CO}_{2}$. Agr Forest Meteorol 174: 144-57.

Fuchslueger L, Bahn M, Fritz K, et al. 2014. Experimental drought reduces the transfer of recently fixed plant carbon to soil microbes and alters the bacterial community composition in a mountain meadow. New Phytol 201: 916-27.

Grünzweig JM and Körner C. 2001. Growth, water and nitrogen relations in grassland model ecosystems of the semi-arid Negev of Israel exposed to elevated $\mathrm{CO}_{2}$. Oecologia 128: 251-62.

Gutschick VP and BassiriRad H. 2003. Extreme events as shaping physiology, ecology, and evolution of plants: toward a unified definition and evaluation of their consequences. New Phytol 160: 21-42.

Hartmann H, Ziegler W, Kolle O, et al. 2013. Thirst beats hunger declining hydration during drought prevents carbon starvation in Norway spruce saplings. New Phytol 200: 340-49.

Hättenschwiler S, Miglietta F, Raschi A, et al. 1997. Thirty years of in situ tree growth under elevated $\mathrm{CO}_{2}$ : a model for future forest responses? Glob Change Biol 3: 463-71.

Hoeppner SS and Dukes JS. 2012. Interactive responses of old-field plant growth and composition to warming and precipitation. Glob Change Biol 18: 1754-68.

Hoover DL, Knapp AK, and Smith MD. 2014. Resistance and resilience of a grassland ecosystem to climate extremes. Ecology 95: 2646-56.

Hueve K, Bichele I, Rasulov B, et al. 2011. When it is too hot for photosynthesis: heat-induced instability of photosynthesis in relation to respiratory burst, cell permeability changes and $\mathrm{H}_{2} \mathrm{O}_{2}$ formation. Plant Cell Environ 34: 113-26.

Jones SE and Lennon JT. 2010. Dormancy contributes to the maintenance of microbial diversity. P Natl Acad Sci USA 107: 5881-86.

Knapp AK, Beier C, Briske DD, et al. 2008. Consequences of more extreme precipitation regimes for terrestrial ecosystems. BioScience 58: 811-21.

Larcher W, Kainmüller C, and Wagner J. 2010. Survival types of 
high mountain plants under extreme temperatures. Flora 205 3-18.

Lenton TM. 2011. Early warning of climate tipping points. Nature Climate Change 1: 201-09.

McDowell NG. 2011. Mechanisms linking drought, hydraulics, carbon metabolism, and vegetation mortality. Plant Physiol 155: 1051-59.

McDowell NG, Beerling DJ, Breshears DD, et al. 2011. The interdependence of mechanisms underlying climate-driven vegetation mortality. Trends Ecol Evol 26: 523-32.

McDowell NG, Fisher RA, Xu C, et al. 2013. Evaluating theories of drought-induced vegetation mortality using a multimodelexperiment framework. New Phytol 200: 304-21.

Mora C, Frazier AG, Longman RJ, et al. 2013. The projected timing of climate departure from recent variability. Nature 502 : 183-87.

Paul ND. 2000. Stratospheric ozone depletion, UV-B radiation and crop disease. Environ Pollut 108: 343-55.

Sagarin R and Pauchard A. 2009. Observational approaches in ecology open new ground in a changing world. Front Ecol Environ 8: 379-86.

Scheffer M and Carpenter SR. 2003. Catastrophic regime shifts in ecosystems: linking theory to observation. Trends Ecol Evol 18: $648-56$.
Sevanto S, McDowell NG, Dickman LT, et al. 2014. How do trees die? A test of the hydraulic failure and carbon starvation hypotheses. Plant Cell Environ 37: 153-61.

Smith MD. 2011. The ecological role of climate extremes: current understanding and future prospects. J Ecol 99: 651-55.

Sowerby A, Emmett BA, Tietema A, and Beier C. 2008. Contrasting effects of repeated summer drought on soil carbon efflux in hydric and mesic heathland soils. Global Change Biol 14: 2388-404.

Tognetti R, Cherubini P, and Innes JL. 2000. Comparative stemgrowth rates of Mediterranean trees under background and naturally enhanced ambient $\mathrm{CO}_{2}$ concentrations. New Phytol 146: 59-74.

${ }^{4}$ Robert H Smith Faculty of Agriculture, Food and Environment, the Hebrew University of Jerusalem, Rehovot, Israel; ${ }^{5}$ Institute for Agricultural Sciences, ETH Zurich, Zurich, Switzerland; ${ }^{6}$ Norwegian Institute for Water Research, Oslo, Norway; ${ }^{7}$ Earth and Environmental Sciences Division, Los Alamos National Laboratory, Los Alamos, NM; ${ }^{8}$ Department of Forestry and Natural Resources and Department of Biological Sciences, Purdue University, West Lafayette, IN

\section{Join us for the 100th ESA Annual Meetingl Sunday August 9-Friday August 14, 2015 Baltimore Convention Center Baltimore, Maryland}

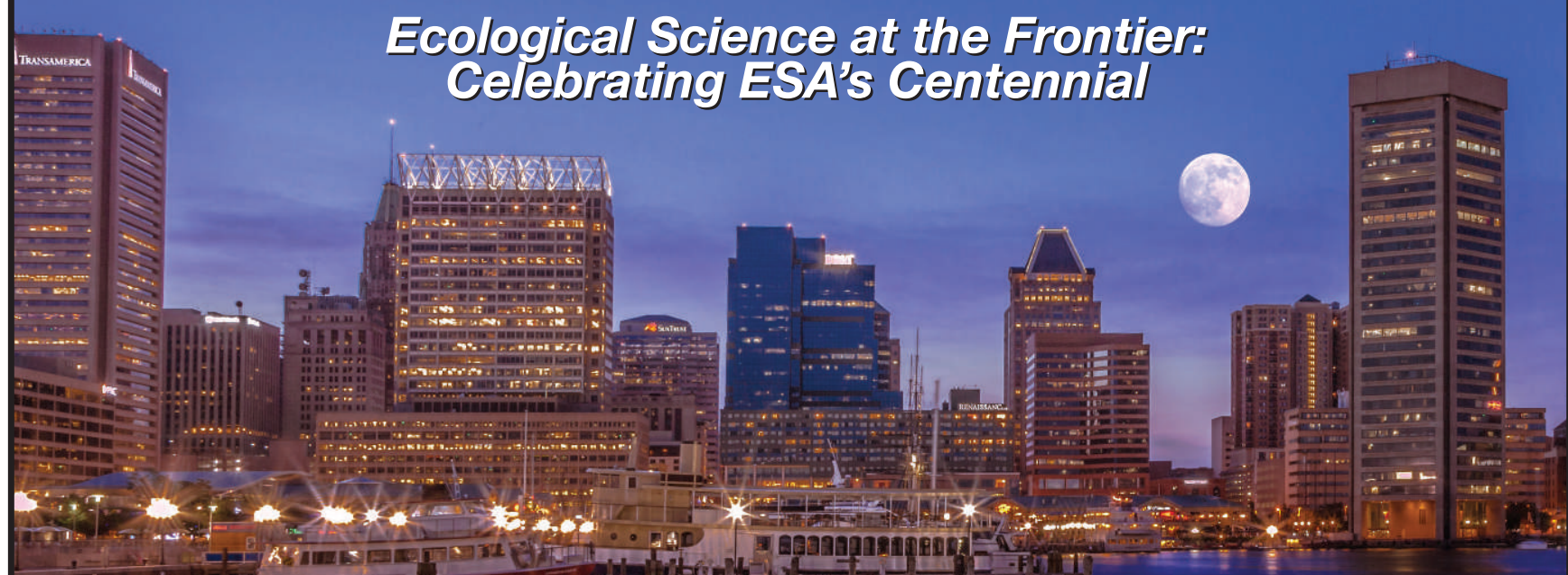

Opening Plenary: The Crossroads Project offers a powerful blend of music, imagery, and science

Centennial Ecologly Lectures will be given by Chris Field, Joshua Tewksbury, and Mercedes Pascual

\section{Don't forget to renew your membership for 2015 to receive reduced registration fees for this very special event!}

For more information visit: wwwesa.org/baltimore 